\title{
V005 TRACE CONTINUITY ANALYSIS FOR STRUCTURE INTERPRETATION - CASE STUDIES ON CARBONATE RESERVOIRS
}

\author{
HIROSHI HAGIWARA', KATUHEI YOSHIOKA² and NOBUSUKE SHIMADA ${ }^{2}$ \\ Japan Oil Development Co. Ltd, Yamato Life Insurance Building 1-1-7 Uchisaiwaicho, Chiyoda-ku, \\ Tokyo 100, Japan
}

\section{Introduction}

In order to define accurate reservoir models for the enhancement of the oil production, a methodology of the structure lineament extractions from the 3 -D post-stack seismic data is becoming more important, not only for exploration, but also for exploitation. Following the increasing use of interpretation software, which works on the powerful workstation system, structure interpretation has been made easier. However, the fault interpretation still has some difficulties. This is because the fault planes are mainly recognized as the discontinuity of the seismic reflectors. In other words, faults have not been revealed as the numerical fault "surface" to date.

The continuity analysis is a method for identifying faults and stratigraphic features on 3-D seismic data volume by measuring the localized waveform similarity or continuity using crosscorrelation techniques with nearest neighbouring traces (Bahorich and Farmer, 1995). This paper describes one of the case studies to examine this method on the heterogeneous carbonate reservoirs.

\section{Methodology}

The continuity analysis is a measurement of the similarity for two waveforms (crosscorrelation). For two waveforms $G_{T}$ and $H_{T}$, the normalized cross-correlation function $\phi$, at time $t$ and $\operatorname{dip} d$ within the time window $\Delta t$, is as follows (Sheriff,1991):

$$
\phi(t, d t)=\frac{\sum_{T=t-\Delta t / 2}^{T=t+\Delta t / 2} G_{T} H_{T+d t}}{\sqrt{\sum_{T=t-\Delta t / 2}^{T=t+\Delta t / 2} G_{T}^{2} \sum_{T=t-\Delta t / 2}^{T=t+\Delta t / 2} H_{T+d t}^{2}}}
$$

The computation is repeated for every possible dip in time $d t$ within the search limits, and the highest correlation coefficient value is adopted for the result of the original trace $G_{T}$. After expanding this calculation for each adjacent trace both inline and crossline directions, a minimum coefficient value is selected as a representative value of the original trace $G_{T}$.

\section{Case study}

This is an example of offshore 3-D seismic data acquired from a domal structure. It has 
been interpreted as a structural trap associated with the salt plug intrusion.

Fig.1 shows an example of the amplitude time slice section at the north extension of the structure. There are some fault-like discontinuities identified extending from the salt body to the frank. After the coherency analysis, the faults are clearly more visible (Fig.2) than when it is observed on the conventional time slice section. This is because the discontinuities in the coherent analysis are revealed as numerically separated surfaces from the laterally continuous area. Fig.3 shows 3-D seismic cube after the continuity analysis. It is clear that the discontinuities are also visible on the vertical seismic sections. Same calculation was done around the salt plug with the wide band dip search limits for dipping events. The result shows low-continuity values zone resulting around the salt plug zone. This is probably because of the lack of the reflector and/or the highly dipping events within the salt body.

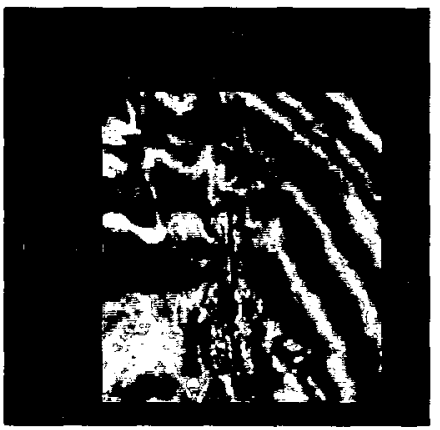

Fig. 1 amplitude time slice
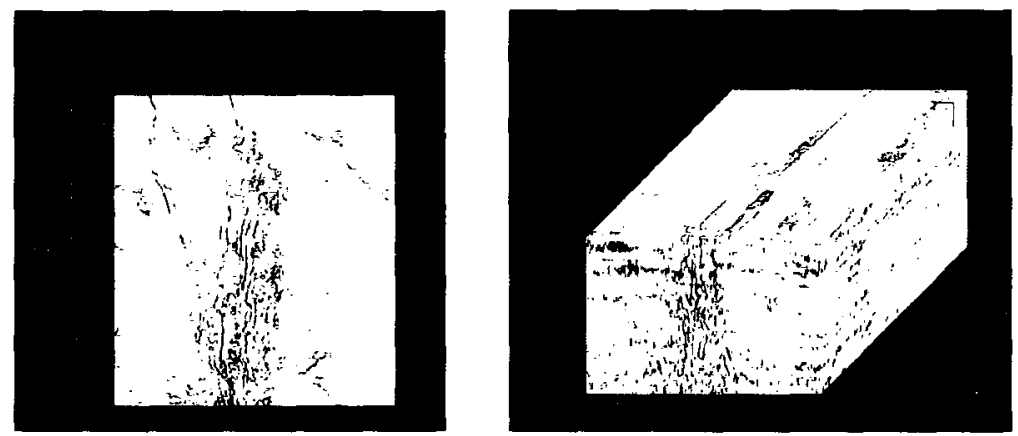

Fig.2 Coherency analysis Fig.3 Coherency analysis (3-D cube)

\section{Conclusion}

The continuity analysis provides an interpreter with a new view of the seismic data without any horizon interpretations alongside time slices and vertical seismic sections. It can be used not only for fault but also for the stratigraphic features delineations, such as salt edges, channels, karsts, pinnacle reefs. The expression of the discontinuity by the numerical values must improve the fault interpretation method on the workstation system. However, it must be noted that the suppression of the acquisition/processing footprint affecting to the wavelet shapes (that are not due to geological/paleotopographical change) is necessary for the coherency analysis. Considerable care must be taken for the input data and the analyzed results.

\section{References}

Bahorich, M.S. and Farmer, S.L., 1995, The coherency cube. The Leading Edge, vol.14, no.10, 1053-1058

Sheriff, R.E., 1991, Encyclopedic dictionary of exploration geophysics, 3rd ed. Tulsa, SEG, $376 \mathrm{p}$ 